Rechtsmedizin 2020 · 30:300-304 https://doi.org/10.1007/s00194-020-00410-3 Online publiziert: 1 . September 2020

(c) Der/die Autor(en) 2020
E. Betschart ${ }^{1}$ A. Dobay' $\cdot$ L. Ebert ${ }^{1}$ - D. Guebelin ${ }^{1} \cdot$ M. J. Thali' $\cdot$ S. Franckenberg ${ }^{1,2}$

${ }^{1}$ Institut für Rechtsmedizin, Universität Zürich, Zürich, Schweiz

${ }^{2}$ Institut für Diagnostische und Interventionelle Radiologie, Universitätsspital Zürich, Zürich, Schweiz

\title{
Einfluss der die Legalinspektion durchführenden Arztgruppen auf die Dauer der Obduktion
}

Die ärztliche Untersuchung einer Leiche ist im europäisch-deutschsprachigen Raum (Deutschland/Schweiz: Leichenschau, Österreich: Toten-/Leichenbeschau) bei jedem Verstorbenen obligatorisch. Sie beinhaltet neben der sicheren Feststellung des Todes, der Schätzung des Todeszeitpunktes sowie der sicheren Feststellung der Identität des Verstorbenen eine Deklaration der Todesart als natürlich, nichtnatürlich oder unklar; diese „normale“ Leichenschau kann prinzipiell durch jeden approbierten Arzt durchgeführt werden [2-4, 6, 9].

Unklare Todesfälle, sowie nichtnatürliche Todesfälle (Unfall, Suizid, Tötungsdelikt, medizinischer Behandlungsfehler) müssen gemeldet werden; die jeweiligen Strafverfolgungsbehörden ordnen dann die Untersuchung des Leichnams durch entsprechend spezialisierte Ärzte (in der Regel Rechtsmediziner) an. In der Schweiz werden diese nichtnatürlichen, auf eine Gewalteinwirkung verdächtigen oder plötzlichen, unerwarteten Todesfälle als Außergewöhnlicher Todesfall (AgT) bezeichnet, und die damit einhergehende spezielle Form der Leichenschau wird Legalinspektion genannt $[6,8,10]$. Die Legalinspektion wird durch einen forensisch ausgebildeten Arzt (Amts-/ Bezirksarzt oder Arzt eines rechtsmedizinischen Instituts; nicht zwingend Facharzt für Rechtsmedizin) durchgeführt, der Untersuchungsablauf am Leichnam wird mit den Anwesenden (Staatsanwaltschaft, Polizei, ggf. Kriminaltechnik u. a.) abgesprochen [5]. Die Untersuchung des Leichnams im forensischen Kontext beinhaltet neben den Kernpunkten der "normalen“ Leichenschau eine wesentlich detailliertere Untersuchung des Leichnams und u. a. genauer Dokumentation des Fundortes sowie der Lage und Kleidung des Leichnams [2, 5]. Je nach Untersuchungsergebnissen wird der Leichnam anschließend durch die zuständige Staatsanwaltschaft freigegeben, eine weiterführende Abklärung mittels Computertomografie-Triage (Modell Zürich), weitere Bildgebungsmodalitäten wie eine Magnetresonanztomographie (MRT) oder ein Oberflächenscan, oder direkt eine Obduktion angeordnet. Anlässlich der Obduktion wird erneut eine äußere Besichtigung des Leichnams durchgeführt; anschließend werden die 3 Körperhöhlen eröffnet, sämtliche Organe beurteilt sowie histologische, Blutund Urinproben asserviert [1,7].

Letztlich gibt es in den meisten forensischen Obduktionsfällen also zwei äuBere Besichtigungen: eine erste vor Ort, von einem Amts-/Bezirksarzt oder von einem Arzt des zuständigen rechtsmedizinischen Instituts durchgeführt, und eine zweite anlässlich der Obduktion am Institut für Rechtsmedizin [7]. Die Sinn- haftigkeit dieser zweiten äußeren Besichtigung ist unbestritten, kann hier doch unter optimierten Untersuchungsbedingungen (Lichtverhältnisse, Hilfsmaterialien, Reinigung etc.) der Leichnam erneut bzw. ergänzend evaluiert werden; sie sollte also unabhängig von der Legalinspektion mit der jeweils gleichen Sorgfalt und Gründlichkeit durchgeführt werden. Da jedoch unterschiedliche Arztgruppen die Legalinspektion durchführen und es dadurch zu einer gewissen Heterogenität dieser Untersuchung kommt, ist die Frage, ob diese Heterogenität für die nachfolgende Fallbearbeitung relevant ist. Der die Obduktion durchführende Rechtsmediziner könnte in seiner äußeren Besichtigung von dem Vorwissen über die Legalinspektion, bzw. wer diese durchgeführt hat, beeinflusst sein - und abhängig davon die äußere Besichtigung der Obduktion unterschiedlich sorgfältig bzw. zeitaufwendig durchführen. So könnte z. B. die Legalinspektion durch einen externen Arzt als ungenügend oder die wiederholte äußere Besichtigung durch einen Rechtsmediziner desselben Insti-
Tab. 1 Aufteilung der Obduktionsfälle nach Todesart, Geschlecht und der die Legalinspektion und die Obduktion durchführenden Arztgruppe

\begin{tabular}{llll} 
& Fälle (weiblich) & $\begin{array}{l}\text { Legalinspektion } \\
\text { LE/LI }\end{array}$ & $\begin{array}{l}\text { Obduktion } \\
\text { LI }_{\mathbf{1}} / \mathbf{L}_{\mathbf{2}}\end{array}$ \\
\hline$n$ & $892(291)$ & $503 / 389$ & $66 / 323$ \\
Natürliches inneres Geschehen & $431(144)$ & $259 / 172$ & $28 / 144$ \\
\hline Unfall & $205(61)$ & $109 / 96$ & $17 / 79$ \\
Selbsttötung & $91(27)$ & $40 / 51$ & $9 / 42$ \\
"Drogentod" & $144(46)$ & $92 / 52$ & $7 / 45$ \\
Tötungsdelikt & $21(13)$ & $3 / 18$ & $5 / 13$
\end{tabular}

LE Amts-/Bezirksarzt, $L$ I Legalinspekteur des Instituts für Rechtsmedizin Zürich, $L I_{1}$ Legalinspekteur und Obduzent sind dieselbe Person, $L I_{2}$ Legalinspekteur und Obduzent sind nicht dieselbe Person 


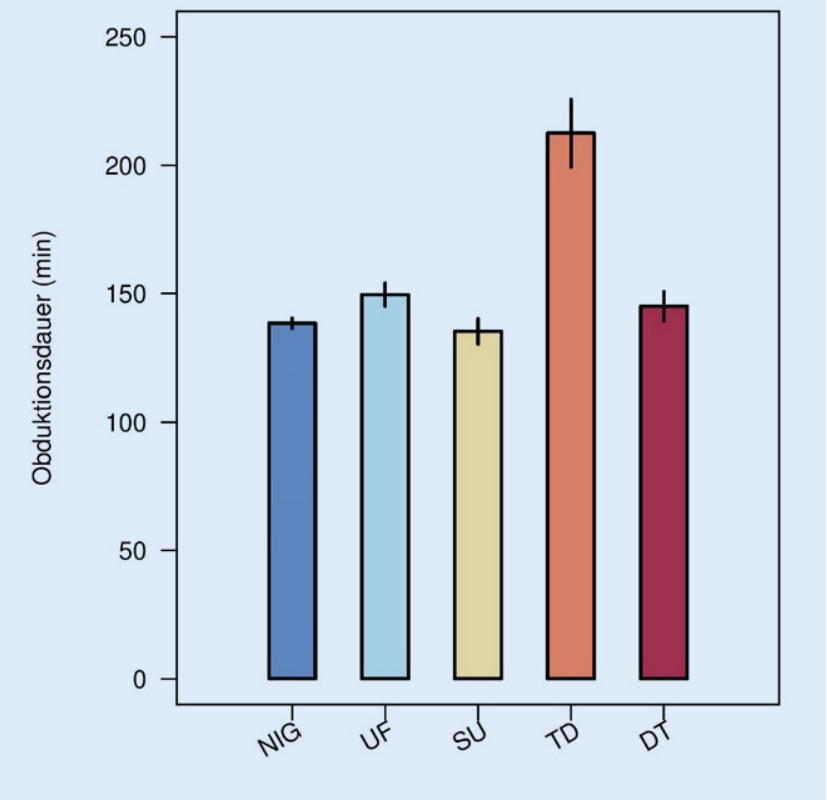

\section{Ergebnisse}

Eine Übersicht der Falldaten ist in • Tab. 1 dargestellt.

Die durchschnittliche Obduktionsdauer für jede Todesart (unabhängig von der die Legalinspektion durchführenden Arztgruppe) ist in - Abb. 1 dargestellt. Während es zwischen einem natürlichen inneren Geschehen, Unfall, Selbsttötung und „Drogentod“ keine wesentlichen Unterschiede in der Obduktionsdauer gibt, ist die Zeit im Falle der Tötungsdelikte erwartungsgemäß deutlich länger.

Die - Abb. 2a-e zeigt die Darstellung
Abb. $1<$ Durchschnittliche Obduktionsdauer abhängig von der Todesart. NIG natürliches inneres Geschehen, UFUnfall, SU Suizid, TD Tötungsdelikt, DTDrogentod tuts als Redundanz angesehen werden, insbesondere dann, wenn der obduzierende Rechtsmediziner die vorherige Legalinspektion selbst durchgeführt hat.

Wir untersuchten daher die Hypothesen (I), dass es einen Unterschied in der Obduktionsdauer abhängig von der die Legalinspektion durchführenden Arztgruppe gibt und (II) dass die Obduktionszeit am kürzesten ist, wenn der die Legalinspektion und die Obduktion durchführende Arzt derselbe ist.

\section{Methodik}

Für diese retrospektive Studie untersuchten wir die Obduktionsfälle am Institut für Rechtsmedizin der Universität Zürich zwischen Januar 2017 und Juni 2019 $(n=1102)$. Ausgeschlossen wurden alle Obduktionsfälle, bei denen die Todesart nicht eindeutig bestimmt werden konnte $(n=158)$, die im Kontext eines möglichen medizinischen Behandlungsfehlers standen $(n=11)$, und Fälle ohne vorherige Legalinspektion $(n=41)$. Das Studienkollektiv umfasste letztlich 892 Fälle (davon weiblich 291, entsprechend 32,6\%; Durchschnittsalter 52 Jahre; Standardabweichung 44 bis 56 Jahre). Informationen zur Legalinspektion und Obduktion (demografische Daten, verantwortlicher Arzt, Dauer der Obduktion [Gesamtzeit aus innerer und äußerer Besich- tigung], Todesursache und -art) wurden den archivierten Berichten entnommen. Die Obduktionen wurden standardmäßig immer von zwei Ärzten durchgeführt. Aufgrund des vorhersehbaren unterschiedlichen zeitlichen Aufwands einer Obduktion in Abhängigkeit von der vermuteten Todesart erfolgte eine Unterteilung der Fälle in vier Subgruppen gemäß der in der Schweiz üblichen Nomenklatur in natürliches inneres Geschehen (NIG, in Deutschland: natürlicher Tod, NT), Unfall (UF), Suizid (SU) und Tötungsdelikt (TD, D: Homizid, HO). Die große Gruppe fataler Intoxikationen, bei denen nicht zwischen einem Unfall und Suizid unterschieden werden konnte, wurde neben den oben aufgeführten Todesarten in einer separaten Kategorie als „Drogentod“ (DT) erfasst. Die Arztgruppen, die die Legalinspektionen durchführten, wurden in externe Ärzte (Amtsärzte, Bezirksärzte; nachfolgend als Legalinspekteur extern: LE abgekürzt) und interne Ärzte (am Institut für Rechtsmedizin angestellt; nachfolgend Legalinspekteur intern: LI abgekürzt) eingeteilt.

Zur statistischen Analyse wurden One-way-ANOVA-Tests durchgeführt. Als statistisch signifikant wurde ein $p<0,05$ angenommen. der Obduktionsdauer, aufgeteilt nach Todesart und unter Berücksichtigung der die Legalinspektion durchführenden Arztgruppe (LE, $\mathrm{LI}_{1}$ oder $\mathrm{LI}_{2}$ ). In Bezug auf unsere Fragestellung zeigt sich im One-way-ANOVA-Test für keine der Todesarten ein signifikanter Unterschied $(p>0,05)$ der Obduktionsdauer in Abhängigkeit des Legalinspekteurs (Amts-/Bezirksarzt vs. Arzt des Instituts für Rechtsmedizin Zürich). Ebenfalls keinen signifikanten Unterschied der Obduktionsdauer ergibt sich, wenn Legalinspekteur und Obduzent dieselbe, respektive nicht dieselbe Person sind.

\section{Diskussion}

Unsere Daten zeigen, dass es keine statistisch signifikante Korrelation zwischen der Obduktionsdauer (in Subgruppen zur Todesart) und den die Legalinspektion durchführenden Arztgruppen (Amts-/Bezirksarzt vs. Ärzte des Instituts für Rechtsmedizin) gibt; somit kann unsere erste Hypothese verworfen werden, dass es einen Unterschied in der Obduktionsdauer abhängig von der die Legalinspektion durchführenden Arztgruppe gibt. Ebenfalls verworfen werden kann unsere zweite Hypothese, dass die Obduktionsdauer am kürzesten sei, wenn Legalinspekteur und Obduzent dieselbe Person sind.

Daraus schließen wir, dass die Legalinspektion und die anlässlich der Obduktion durchgeführte, „zweite“ äußere Besichtigung tatsächlich als zwei voneinander völlig unabhängige Untersuchungen wahrgenommen und gehandhabt werden. Selbst wenn Obduzent und Legal- 
Rechtsmedizin 2020 · 30:300-304 https://doi.org/10.1007/s00194-020-00410-3

(c) Der/die Autor(en) 2020

E. Betschart · A. Dobay · L. Ebert · D. Guebelin · M. J. Thali · S. Franckenberg

\section{Einfluss der die Legalinspektion durchführenden Arztgruppen auf die Dauer der Obduktion}

Zusammenfassung

Hintergrund. Die Legalinspektion, als Schweizer Begriff für die Leichenschau im forensischen Kontext, wird entweder von einem Amts-/Bezirksarzt oder einem an einem rechtsmedizinischen Institut angestellten Arzt durchgeführt. Durch diese unterschiedlichen Arztgruppen kommt es zwangsläufig zu einer gewissen Heterogenität der Untersuchung. Fragestellung. Wir untersuchten den Einfluss von der die Legalinspektion durchführenden Arztgruppe (Amts-/Bezirksarzt und Arzt des Rechtsmedizinischen Instituts Zürich) auf die Obduktionsdauer. Der die Obduktion durchführende Rechtsmediziner könnte in seiner äußeren Besichtigung von dem Vorwissen über die Legalinspektion, bzw. wer diese durchgeführt hat, beeinflusst sein und abhängig davon die äußere Besichtigung der Obduktion unterschiedlich sorgfältig bzw. zeitaufwendig durchführen. Es wurden zwei Hypothesen überprüft: zum einen, dass es einen Unterschied der Obduktionsdauer abhängig von der die Legalinspektion durchführenden Arztgruppe gibt, zum anderen, dass die Obduktionszeit am kürzesten ist, wenn der die Legalinspektion und die Obduktion durchführende Arzt derselbe ist. Methodik. Wir führten eine retrospektive Analyse an 892 konsekutiven Obduktionsfällen am Institut für Rechtsmedizin der Universität Zürich durch (Januar 2017 bis Juni 2019). Für jeden Fall wurden die die Legalinspektion durchführende Arztgruppe sowie die Obduktionszeit aus den archivierten Berichten erhoben. Anschließend wurden die erhobenen Variablen in Untergruppen nach Todesart miteinander verglichen (One-wayANOVA-Tests).

Ergebnisse. Die Obduktionsdauer zeigte keinen signifikanten Unterschied in allen Todesartsubgruppen zwischen den die Legalinspektion durchführenden Arztgruppen. Selbst wenn Legalinspekteur und Obduzent dieselbe Person waren, unterschied sich die
Obduktionszeit nicht signifikant von den anderen Arztgruppen. Somit waren beide Hypothesen zu verwerfen.

Diskussion. Legalinspektion und Obduktion werden als zwei unabhängige Untersuchungen wahrgenommen. Die "zweite" äußere Besichtigung wird selbst dann nicht als redundant empfunden, wenn Obduzent und Legalinspekteur dieselbe Person sind. Unsere Ergebnisse sehen wir auch als Ausdruck einer breiten Akzeptanz und guten Implementation der Leitlinien für Legalinspektion und Obduktion mit klar definierten Anforderungen und standardisierten Abläufen. Weiterführende Studien mit der Erfassung anderer und ergänzender Parameter zur Beurteilung auch der inhaltlichen Qualität der Legalinspektion und Obduktion sind sinnvoll und notwendig.

Schlüsselwörter

Amtsarzt $\cdot$ Rechtsmediziner $\cdot$ Qualitätssicherung $\cdot$ Bezirksarzt $\cdot$ Zürich

\section{Influence of the groups of doctors conducting the medico-legal inspection on the duration of the autopsy}

\section{Abstract}

Background. Medico-legal examinations on site, a Swiss term for the external examination of a corpse, are performed by either public health officers or forensic pathologists associated with an institute of forensic medicine in Switzerland. These different types of physicians performing the examination lead to a certain degree of heterogeneity in the investigation.

Objective. We investigated the influence of the type of physician performing the medico-legal examination on site (public health officer and forensic pathologist from the Institute of Forensic Medicine, University of Zurich) on the overall autopsy duration. The physician performing the autopsy might be influenced by knowledge about which physician performed the medico-legal examination on site and therefore execute the external examination at autopsy with a different thoroughness or duration. We tested two hypotheses. First, that there is a difference in overall autopsy duration depending on the type of physician performing the medico-legal examination on site. Second, that autopsy duration is shortest if the same physician performs the medico-legal examination on site and the subsequent autopsy.

Methods. We performed a retrospective analysis of 892 consecutive autopsy cases at the Institute of Forensic Medicine, University of Zurich. For each case, the type of physician performing the external examination on site and the overall duration of the autopsy were retrieved from the archives. These variables were compared in subgroups according to the manner of death (one-way ANOVA tests). Results. The overall autopsy duration showed no significant differences in all manner of death subgroups for the type of physician performing the medico-legal examination on site. Both our hypotheses can therefore be rejected.
Conclusion. The medico-legal examination on site and autopsy seem to be regarded as two independent examinations. The second external examination is not seen as redundant, even if the physician performing the autopsy is the same person who performed the previous medico-legal examination on site. Our results can be interpreted as an expression of broad acceptance and good implementation of the guidelines for medicolegal examinations and autopsies, with clearly defined requirements and standardized processes. Further studies with the evaluation of other and adjunct parameters with respect to the content-related quality of the medicolegal examination and autopsy make sense and are necessary.

Keywords

Public health officer - Forensic pathologist . Quality assurance · District physician · Zurich 

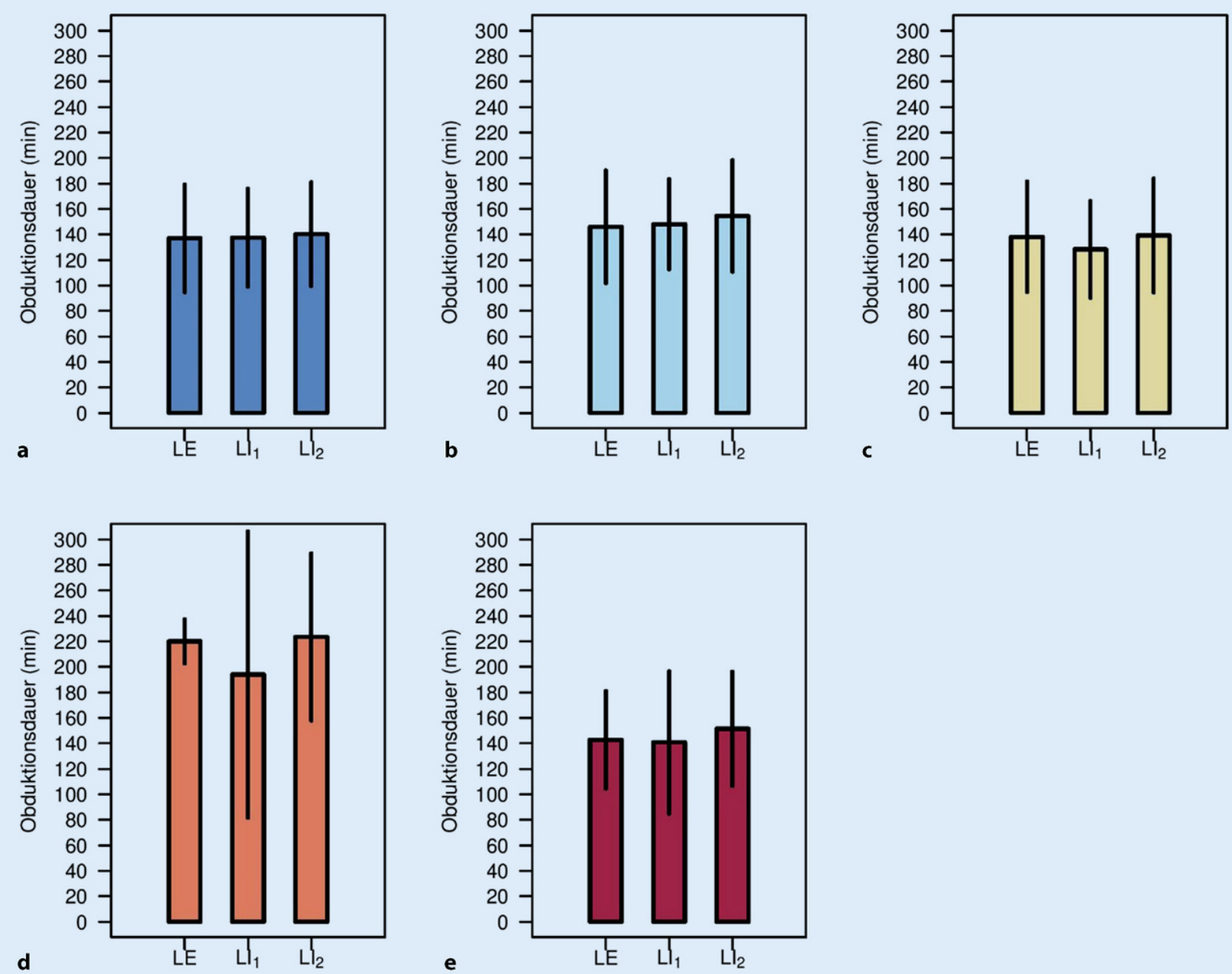

Abb. $2 \Delta$ Obduktionsdauer in Abhängigkeit von der die Legalinspektion durchführenden Arztgruppe für die Todesarten a natürliches inneres Geschehen, b Unfall, c Suizid, d Tötungsdelikt und e „Drogentod". LE Amts-/Bezirksarzt, LI 1 Legalinspekteur und Obduzent sind dieselbe Person, $L L_{2}$ Legalinspekteur und Obduzent sind nicht dieselbe Person

inspekteur dieselbe Person sind, kommt es zu keiner Verkürzung der durch sie „doppelt“ durchgeführten äußeren Besichtigung, die somit offensichtlich auch nicht als redundant empfunden wird. Unsere Ergebnisse sehen wir auch als Ausdruck einer breiten Akzeptanz und guten Implementation der Leitlinien für Legalinspektion und Obduktion, mit klar definierten Anforderungen und standardisierten Abläufen [5, 7].

Eine Limitation unserer Studie ist, dass unsere Studiengruppe das Einzugsgebiet nur eines rechtsmedizinischen Instituts der Schweiz widerspiegelt und nicht repräsentativ für die gesamte Schweiz gesehen werden kann. Des Weiteren wurde die Zeitdauer der Obduktion nicht in äußere und innere Besichtigung unterteilt, was für Folgestudien zu berücksichtigen wäre. Außerdem lässt der bloße Vergleich der Obduktionsdauer in Abhängigkeit der die Legalinspektion durchführenden Arztgruppe natürlich keinen eigentlichen Rückschluss auf die inhaltliche Qualität der Untersuchung zu. Dies müsste mit anderen Parametern, z. B. mit der inhaltlichen Analyse der Legalinspektionsberichte, erfasst werden. Des Weiteren wird die Dauer der Legalinspektionen bei keiner der Arztgruppen erfasst, sodass wir diesen zeitlichen Parameter nicht zusätzlich in unsere Auswertung integrieren konnten (z. B. im direkten Vergleich mit der Zeitdauer der äußeren Besichtigung anlässlich der Obduktion).

\section{Ausblick}

Unsere Daten scheinen einen einheitlichen Prozessablauf, unabhängig von der vorher erfolgten Legalinspektion, in der äußeren Besichtigung anlässlich der Obduktion zu bestätigen, auch wenn unsere Ergebnisse und Interpretationen angesichts der oben genannten Limitationen mit einem gewissen Vorbehalt zu betrachten sind.

Insbesondere in Anbetracht zunehmend geforderter Qualitätssicherungen und Zertifizierungsprozesse in der Rechtsmedizin sind weiterführende Stu- 
dien mit der Erfassung anderer und ergänzender Parameter sinnvoll und notwendig.

\section{Fazit für die Praxis}

- Das Wissen darum, welche Arztgruppe die Legalinspektion durchgeführt hat, beeinflusst nicht die Dauer der Obduktion.

- Legalinspektion und äußere Besichtigung der Obduktion werden als zwei voneinander unabhängige Untersuchungen wahrgenommen und gehandhabt.

- Die Leitlinien für Legalinspektion und Obduktion scheinen weitläufig akzeptiert und gut implementiert zu sein.

- In Anbetracht zunehmend geforderter Qualitätssicherungen und Zertifizierungsprozesse in der Rechtsmedizin sind weiterführende Studien sinnvoll und notwendig.

\section{Korrespondenzadresse}

\section{med. pract. E. Betschart}

Institut für Rechtsmedizin, Universität Zürich Winterthurerstr. 190/52, 8057 Zürich, Schweiz eva.betschart@uzh.ch

Funding. Open access funding provided by University of Zurich

\section{Einhaltung ethischer Richtlinien}

Interessenkonflikt. E. Betschart, A. Dobay, L. Ebert, D. Guebelin, M.J. Thali und S. Franckenberg geben an, dass kein Interessenkonflikt besteht.

Für diesen Beitrag wurden von den Autoren keine Studien an Menschen oder Tieren durchgeführt. Für die aufgeführten Studien gelten die jeweils dort angegebenen ethischen Richtlinien. Dieses Forschungsprojekt fällt nicht in den Geltungsbereich des Humanforschungsgesetzes (Nichtzuständigkeitserklärung der Ethikkommission).

Open Access. Dieser Artikel wird unter der Creative Commons Namensnennung 4.0 International Lizenz veröffentlicht, welche die Nutzung, Vervielfältigung, Bearbeitung, Verbreitung und Wiedergabe in jeglichem Medium und Format erlaubt, sofern Sie den/die ursprünglichen Autor(en) und die Quelle ordnungsgemäß nennen, einen Link zur Creative Commons Lizenz beifügen und angeben, ob Änderungen vorgenommen wurden.

Die in diesem Artikel enthaltenen Bilder und sonstiges Drittmaterial unterliegen ebenfalls der genannten
Creative Commons Lizenz, sofern sich aus der Abbildungslegende nichts anderes ergibt. Sofern das betreffende Material nicht unter der genannten Creative Commons Lizenz steht und die betreffende Handlung nicht nach gesetzlichen Vorschriften erlaubt ist, ist für die oben aufgeführten Weiterverwendungen des Materials die Einwilligung des jeweiligen Rechteinhabers einzuholen.

Weitere Details zur Lizenz entnehmen Sie bitte der Lizenzinformation auf http://creativecommons.org/ licenses/by/4.0/deed.de.

\section{Literatur}

1. Deutsche Gesellschaft Für Rechtsmedizin (2017) Die rechtsmedizinische Leichenöffnung. In: Leitlinien der Deutschen Gesellschaft für Rechtsmedizin. AWMF online, S. 1-16. https://www.awmf.org/ leitlinien/detail/II/054-001.html. Zugegriffen: 23. Juli 2020

2. Deutsche Gesellschaft Für Rechtsmedizin (2017) Regeln zur Durchführung der ärztlichen Leichenschau. In: Leitlinien der Deutschen Gesellschaft für Rechtsmedizin. AWMF online, S. 1-12. https:// www.awmf.org/leitlinien/detail/ll/054-002.html Zugegriffen: 23. Juli 2020

3. Madea B (2015) Leichenschau - Feststellung der Todesursache und Qualifikation der Todesart. In: Madea B (Hrsg) Rechtsmedizin-Befunderhebung, Rekonstruktion, Begutachtung, 3. Aufl. Springer, Berlin Heidelberg, S50-60

4. Madea B (2015) Leichenschau - Feststellung des Todes. In: Madea B (Hrsg) Rechtsmedizin Befunderhebung, Rekonstruktion, Begutachtung, 3. Aufl. Springer, Berlin Heidelberg, S45-50

5. Arbeitsgruppe Qualitätsmanagement in der Forensischen Medizin. Schweizerische Gesellschaft für Rechtsmedizin (2009) Legalinspektion - Praktische Vorgehensweise. https://www.sgrm.ch/ inhalte/Forensische-Medizin/Durchfuehrung Legalinspektion_01.pdf.Zugegriffen:23. Juli 2020 (S. 11-22)

6. Arbeitsgruppe Qualitätsmanagement in der Forensischen Medizin. Schweizerische Gesellschaft für Rechtsmedizin (2009) Legalinspektion Theoretische Grundlagen. https://www.sgrm.ch/ inhalte/Forensische-Medizin/Durchfuehrung Legalinspektion_01.pdf.Zugegriffen: 23. Juli 2020 (S.5-10)

7. Schweizerische Gesellschaft Für Rechtsmedizin (2016) Obduktion. In: Rechtsmedizin von A-Z. https://www.sgrm.ch/de/allgemein/ueber-dierechtsmedizin/rechtsmedizin-von-a-z/. Zugegriffen: 23 . Juli 2020

8. Schweizerische Strafprozessordnung (2007) Untersuchung an Leichen - Aussergewöhnliche Todesfälle. In: StPO, 6. Abschnitt, Art. 253. Die Bundesversammlung der Schweizerischen Eigenossenschaft, Bern. https://www.admin.ch/opc/ de/classified-compilation/20052319/index.html. Zugegriffen: 23. Juli 2020

9. Zentrum Für Gerichtsmedizin MUW (2020) Totenbeschau. https://www.meduniwien.ac.at/ $\mathrm{hp} /$ gerichtsmedizin/allgemeine-informationen/ einfuehrung/totenbeschau/. Zugegriffen: 23. Jul 2020

10. Zollinger U (2015) Rechtslage in der Schweiz. In: Madea B (Hrsg) Rechtsmedizin-Befunderhebung, Rekonstruktion, Begutachtung, 3. Aufl. Springer, Berlin Heidelberg, S 161-165

\section{Fortbildungen für Autoren und Gutachter}

Die ersten Veröffentlichungen sind für jeden Mediziner ein wichtiger Schritt in seiner Karriere als Wissenschaftler. Wissenschaftliche Artikel sind entscheidend dafür, dass die eigene Arbeit in der Community wahrgenommen wird. Es geht darum, die eigenen Ideen national und international auszutauschen und sicherzustellen, dass die Ergebnisse Wirkung erzielen. Die Online-Kurse der Autorenwerkstatt helfen, sich leicht einen Überblick über das Schreiben, Einreichen, Begutachten und Veröffentlichen eines Manuskripts zu verschaffen.

5 Online-Kurse zu den wichtigsten Standards des wissenschaftlichen Publizierens:

- Wie verfasse ich ein Manuskript?

- Writing in English für deutschsprachige Autoren

- Wie funktionieren Publikation und Begutachtung?

- Anleitung zur Open-AccessVeröffentlichung

- Leitfaden zur Peer-ReviewBegutachtung

Für alle, die auf SpringerMedizin.de registriert sind!

Jetzt gratis fortbilden unter www.springermedizin.de/ autorenwerkstatt/ 